\title{
Development of Dirofilaria immitis and Dirofilaria repens in Aedes japonicus and Aedes geniculatus
}

\author{
Cornelia Silaghi ${ }^{1 *}$, Relja Beck², Gioia Capelli ${ }^{3}$, Fabrizio Montarsi ${ }^{3}$ and Alexander Mathis ${ }^{1}$
}

\begin{abstract}
Background: The mosquito-borne filarial nematodes Dirofilaria immitis and Dirofilaria repens primarily affect dogs but also cats, causing heartworm disease or subcutaneous dirofilariosis, respectively, and both may also cause zoonotic diseases in humans. Several mosquito species have been reported as competent vectors for these nematodes, but no data are available for the invasive mosquito species Aedes japonicus (Theobald, 1901). The objective of this study was to describe the development of both D. immitis and D. repens under standardised experimental laboratory conditions in mosquitoes.

Methods: For this purpose, both a laboratory strain and field-collected individuals of the invasive mosquito species Ae. japonicus and, for comparative purposes, a laboratory strain of Aedes geniculatus, a rare indigenous species sharing habitats with Ae. japonicus, and of the tropical species Aedes aegypti were used. Anticoagulated microfilariaemic blood was fed at a density of $3000 \mathrm{mf} / \mathrm{ml}$ to mosquitoes with a hemotek system. Blood-fed mosquitoes were incubated at $27^{\circ} \mathrm{C}$ and $85 \%$ relative humidity, and specimens were dissected under the microscope at pre-set time points to observe developmental stages of both Dirofilaria species. Additionally, real-time PCRs were carried out in some microscopically negative samples to determine the infection rates.

Results: In field-collected Ae. japonicus infectious $L 3$ larvae of both D. immitis and D. repens developed, rendering this mosquito species an efficient vector for both filarial species. Additionally, Ae. geniculatus was shown to be an equally efficient vector for both filarial species. Aedes japonicus mosquitoes from a laboratory colony were refractory to $D$. immitis but susceptible to $D$. repens, whereas Ae. aegypti was refractory to both filarial species.

Conclusions: To our knowledge, Aedes japonicus was for the first time shown to be an efficient vector for both $D$. immitis and $D$. repens, indicating that this invasive and locally highly abundant species may contribute to a transmission of filarial worms. The data emphasize the necessity to perform vector competence studies with local mosquito populations as basis for risk assessments. We further demonstrated that detection of filarial DNA in a mosquito species alone does not allow to draw reliable conclusions with regard to its vector competence.
\end{abstract}

Keywords: Dirofilaria immitis, Dirofilaria repens, Aedes japonicus, Aedes geniculatus, Aedes aegypti, Microfilariae, Dog, Vector, Intermediate host

\footnotetext{
* Correspondence: cornelia.silaghi@uzh.ch

${ }^{1}$ National Centre for Vector Entomology, Institute of Parasitology, Vetsuisse

Faculty, University of Zurich, Zurich, Switzerland

Full list of author information is available at the end of the article
}

\section{Ciomed Central}

(c) The Author(s). 2017 Open Access This article is distributed under the terms of the Creative Commons Attribution 4.0 International License (http://creativecommons.org/licenses/by/4.0/), which permits unrestricted use, distribution, and reproduction in any medium, provided you give appropriate credit to the original author(s) and the source, provide a link to the Creative Commons license, and indicate if changes were made. The Creative Commons Public Domain Dedication waiver (http://creativecommons.org/publicdomain/zero/1.0/) applies to the data made available in this article, unless otherwise stated. 


\section{Background}

The mosquito-borne filarial nematodes Dirofilaria immitis (Leidy, 1856) and Dirofilaria repens (Railliet \& Henry, 1911) primarily affect dogs but also cats, causing cardiopulmonary (heartworm disease) or subcutaneous dirofilariosis, respectively. Both filarial worms may also cause zoonotic diseases in humans, in the form of pulmonary (D. immitis) or subcutaneous/ocular (D. repens) dirofilariosis $[1,2]$. Both species have expanded their distribution range in the recent past $[1,3]$. Dirofilaria immitis is endemic globally in regions with tropical or subtropical climates, whereas $D$. repens is restricted to the Old World. Recent detections of DNA of the canine heartworm in mosquitoes [4-6] in temperate climate areas in central Europe as well as climate analyses [4, 7-9] suggest a northward spread. Dirofilaria repens seems to be already endemic in central Europe, based on several DNA detections in mosquito populations [5, 10-12] and a growing number of autochthonous cases in both dogs and humans [13-20].

The transmission of the filarial worms is dependent on the availability of microfilariaemic hosts, competent mosquito vectors and suitable temperatures for the development of the infectious stages in the mosquito [8]. A major driver for the range expansion of canine dirofilarioses is the transport of infected animals from endemic to new areas, e.g. via the import of dogs from Mediterranean countries to central Europe where several mosquito species occur which have a demonstrated vector competence for Dirofilaria spp. [21]. Transmission is feasible in regions where suitable temperatures allow the development of the microfilariae ( $\mathrm{mf}$ ) to the infectious third larval stage (L3) which migrate to the proboscis of the mosquito. This extrinsic development is possible above $14{ }^{\circ} \mathrm{C}$ and is completed when the sum of the daily average degrees above this threshold value has reached at least 130. This value was initially termed 'heartworm development unit' (HDU) but later adapted to 'Dirofilaria development unit' (DDU) due to similar temperature requirements for both Dirofilaria species [22, 23]. Thus, the extrinsic development takes e.g. $10-12$ days at $24-26{ }^{\circ} \mathrm{C}$ but as long as 29 days at $18{ }^{\circ} \mathrm{C}$. Successful transmission of filariae to a host requires an infected mosquito to survive longer than the duration of the extrinsic incubation period. Assuming a maximal life span of 30 days for a mosquito, models revealed that summer temperatures allow the development of L3 at latitudes of $50^{\circ} \mathrm{N}$ in Europe [23]. Indeed, a canine autochthonous case of $D$. immitis dirofilariosis was observed at $54^{\circ} \mathrm{N}$ [24].

In addition, during recent decades, invasive containerbreeding aedine mosquito species have been recorded in areas of Europe [25], and they might contribute to an increased transmission risk of Dirofilaria spp. as evidenced for the Asian tiger mosquito Aedes albopictus
(Skuse, 1894) (= Stegomyia albopicta) in Italy [26, 27]. Local populations of another invasive species, Aedes koreicus (Edwards, 1917) (= Hulecoeteomyia koreica), have been recorded in few instances [28-30], and this species was shown in an experimental study to allow the development of the infectious L3 larvae of D. immitis [31].

No data with regard to vector competence for Dirofilaria spp. are available for a third invasive mosquito species, Aedes japonicus (Theobald, 1901) (= Hulecoeteomyia japonica). This East Asian native mosquito has in recent years invaded large parts of North America and many countries in Europe [32, 33], and it is further expanding [34, 35] (for updated European maps see www.ecdc.europa.eu).

The objectives of this study were to describe the development of both $D$. immitis and D. repens under standardised experimental laboratory conditions in both a laboratory strain and in field-collected individuals of the invasive mosquito species Ae. japonicus. For comparative purposes, these experiments were also done with each a laboratory strain of Aedes geniculatus (Olivier, 1791) (= Dahliana geniculata), a rare species in the Palaearctic Region sharing habitats with Ae. japonicus, and of the tropical species Aedes aegypti (L.) (= Stegomyia aegypti).

\section{Methods \\ Microfilariae inoculum}

Presence, vitality and number of microfilariae in all samples obtained from dogs (see below) were confirmed by microscopy. Briefly, $20 \mu \mathrm{l}$ of blood were mixed with $40 \mu \mathrm{l}$ of distilled water, covered with a cover slide, and microfilariae were counted by examination with a microscope under 100× magnification. Microfilaraemiae of the dogs were determined as average from three counts. Blood was anticoagulated with EDTA or heparin.

\section{Dirofilaria immitis}

Microfilariaemic blood samples from experimentally infected dogs (field isolate from northern Italy) as well as blood from uninfected dogs were kindly provided by Christian Epe (Elanco, St. Aubin, Switzerland).

\section{Dirofilaria repens}

Blood samples were from a dog naturally infected with $D$. repens (selected by one of the authors, RB). The infected dog was a mixed breed, 4.5 year-old and had never left the Daruvar region in north-eastern Croatia. The dog had first been diagnosed positive for microfilariae 2 years prior to the experiments. He was regularly checked by a local veterinarian and was always clinically unremarkable (nice fur, healthy skin, no nodules observed). The infection with $D$. repens was confirmed on DNA from the blood samples by a conventional PCR [36]. No co-infections with other filariae that are covered 
with this diagnostic approach (Acanthocheilonema reconditum, Acanthocheilonema, Dracunculoides spp., Brugia pahangi, Brugia malayi, D. immitis) were detected.

\section{Mosquitoes and inoculation Field-collected Aedes japonicus}

Host-seeking female Ae. japonicus were collected in a forest area within the urban borders of the city of Zurich, using mouth aspirators with four persons ("human baits"). Dry ice was additionally used as attractant. Mosquitoes were transferred into a cylindrical $500 \mathrm{ml}$ plastic cage with moist cotton wool and were taken to the laboratory within $1 \mathrm{~h}$ of collection in the field for oral inoculation.

\section{Laboratory strains}

Laboratory colonies of Ae. japonicus (Pennsylvania strain, PEN) and Ae. aegypti (IPNC) were reared and maintained in a climate chamber in an insectarium under standard laboratory conditions at a temperature of $24{ }^{\circ} \mathrm{C}$ (Ae. japonicus) or $27{ }^{\circ} \mathrm{C}$ (Ae. aegypti), a relative humidity (rh) of $85 \%$ and a light-dark cycle of $16: 8 \mathrm{~h}$ including dusk/dawn phases of $1 \mathrm{~h}$. A recently established colony of Ae. geniculatus (IPZ) [37] was maintained at room temperature as described. Mosquitoes were provided with $5 \%$ glucose solution and water ad libitum. For reproduction they were either provided a mouse as blood source once a week (Ae. japonicus, Ae. aegypti; approved by the Cantonal Veterinary Office of Zurich, permission number ZH064/15) or sheep blood (approved by the Cantonal Veterinary Office of Zurich, permission number ZH008/15) using a standard artificial feeding system $\left(\right.$ Hemotek $^{\mathrm{TM}}$, Hemotek Ltd, Lancashire, UK). For the inoculation experiments, females at an age of 5-7 days were chosen.

\section{Oral inoculation of mosquitoes}

Microfilariaemic counts were adjusted to $3000 \mathrm{mf} / \mathrm{ml}$ with blood from uninfected dogs. Sugar was removed and the mosquitoes were allowed to feed through Parafilm ${ }^{\circ}$ membranes for at least $2 \mathrm{~h}$ on $2 \mathrm{ml}$ blood at $37{ }^{\circ} \mathrm{C}$ in a Hemotek ${ }^{\mathrm{TM}}$ system. In order to boost the blood feeding rates of the field-collected mosquitoes, adenosine triphosphate (ATP, final concentration $5 \mathrm{mM}$ ) was added to the blood [38], and $\mathrm{iGu}^{\circ}$ lure disks (Combi FRC 3003, Silva GmbH \& Co. KG, Lübeck, Germany) were displayed. Mosquitoes that did not take a full blood meal were discarded.

\section{Maintenance of the mosquitoes after inoculation}

Mosquitoes were kept for up to 21 days in a double containment (inner cage $17 \times 17 \mathrm{~cm}$, outer cage $32.5 \times$ $32.5 \mathrm{~cm}$, both Bugdorm, MegaView Science, Taichung,
Taiwan) in an incubator at $27{ }^{\circ} \mathrm{C} / 85 \%$ rh with access to $5 \%$ glucose and water ad libitum.

\section{Dirofilaria detection in mosquitoes \\ Microscopic investigation}

Mosquitoes were anaesthetised by brief exposure to $\mathrm{CO}_{2}$ supplied by dry ice and immobilised by removing their wings and legs on a chill table. Afterwards, they were kept at $4{ }^{\circ} \mathrm{C}$ and dissected individually and immediately before microscopic examination. Malpighian tubules and midgut were separated from the abdomen, and afterwards abdomen, head and thorax were dissected on separate microscopic slides. Clean entomological forceps and sterile needles were used for each dissection. A drop of phosphate buffered saline (PBS) was added onto each slide and the sample carefully covered with a coverslide. Light pressure was applied to facilitate the detection of worms, especially of the early stages of development [39]. All slides were investigated with differential interference contrast microscopy (DIC) with a Leica DM 6000 B microscope at $100-400 \times$ magnification. Photographs of larval stages were taken and their length and width calculated. The Leica software LAS was used for all the analyses.

Within $24 \mathrm{~h}$ of infection, up to three mosquitoes of each group were dissected to assess the number of microfilariae ingested per blood meal. Afterwards, up to three mosquitoes were dissected at fixed days (see below) to investigate the developmental stages of the larvae according to size and morphology $[39,40]$. Freshly dead mosquitoes were also dissected; mosquitoes already dead for a while and desiccated were subjected to PCR analysis only (see below). After microscopic investigation, the slides were rinsed with a few drops of PBS for subsequent PCR analysis.

\section{Molecular analysis}

Mosquito samples with $180 \mu \mathrm{l}$ TE buffer were disrupted in a TissueLyser II (Qiagen, Hilden, Germany) in a $2 \mathrm{ml}$ Eppendorf tube with one $5 \mathrm{~mm}$ stainless steel bead added at 30 beats per second for 1 min with a centrifugation step after $30 \mathrm{~s}$. The samples were incubated with lysis buffer and proteinase $K$ for at least $4 \mathrm{~h}$ or overnight. Afterwards, DNA was isolated using the Qiamp DNA mini kit (Qiagen) according to the manufacturer's instruction. DNA was eluted in $55 \mu \mathrm{l} \mathrm{AE}$ buffer and stored at $-20{ }^{\circ} \mathrm{C}$ until further use.

A real-time PCR targeting the mitochondrial COX 1 gene [41] of $D$. immitis and $D$. repens was designed using GenScript (www.genscript.com/ssl-bin/app/primer). Forward and reverse primers and the Taqman ${ }^{\circ}$ probe were as follows: Diro-f: 5' -GGT GTT TGG GAT TGT TAG TGA A-3'; Diro-r: 5'-CAG CAA TCC AAA TAG AAG CAA-3'; Diro-p: 5'-FAM-TCT GGC CAA 
Table 1 Feeding and mortality rates of Aedes japonicus (laboratory strain PEN; field-collected specimens from Switzerland (CH), Ae. geniculatus (laboratory strain IPZ) and Ae. aegypti (laboratory strain IPNC) during infection trials with Dirofilaria immitis and D. repens

\begin{tabular}{|c|c|c|c|c|c|c|}
\hline Mosquito species & Inoculation & No. total & No. feeding (\%) & $\begin{array}{l}\text { Mortality at } 1 \text { dpi } \\
\text { No. (\%) }\end{array}$ & $\begin{array}{l}\text { Mortality } 5 \text { dpi } \\
\text { No. (\%) }\end{array}$ & $\begin{array}{l}\text { Mortality at } 14 \mathrm{dp} \\
\text { No. (\%) }\end{array}$ \\
\hline Ae. japonicus (PEN) & Dirofilaria repens & 19 & $8(42.1)$ & 0 & 0 & $2(28.6)$ \\
\hline Ae. japonicus (PEN) & D. immitis & 28 & $12(42.3)$ & 0 & $5(50.0)$ & $8(80.0)$ \\
\hline Ae. japonicus (PEN) & Negative control & 18 & $9(50.0)$ & 0 & $3(33.3)$ & $4(44.4)$ \\
\hline Ae. japonicus $(\mathrm{CH})$ & D. repens & 72 & 17 (23.6) & 0 & 0 & 0 \\
\hline Ae. japonicus (CH) & D. immitis & 60 & 31 (51.6) & 0 & $10(35.7)$ & $13(46.4)$ \\
\hline Ae. japonicus (CH) & Negative control & 19 & $8(42.1)$ & 0 & $1(12.5)$ & $5(62.5)$ \\
\hline Ae. geniculatus IPZ & D. repens & 17 & $12(70.1)$ & 0 & 0 & $4(44.4)$ \\
\hline Ae. geniculatus IPZ & D. immitis & 18 & $13(72.2)$ & 0 & $2(18.2)$ & $4(36.4)$ \\
\hline Ae. aegypti IPNC & D. repens & 46 & $21(45.7)$ & $4(22.2)$ & $8(44.4)$ & $8(44.4)$ \\
\hline Ae. aegypti IPNC & D. immitis & 41 & $24(58.5)$ & $6(28.6)$ & $11(52.4)$ & $11(52.4)$ \\
\hline
\end{tabular}

${ }^{\mathrm{a}}$ Only mosquitoes that died naturally; calculated without day 1 samples; numbers are cumulative from day 1

ACA AAC GAT CCT TAT CA-TAMRA-3'. The target size is $98 \mathrm{bp}$. The PCR assay was not evaluated for its diagnostic value.

Real-time PCR was performed in addition to microscopical investigations with microscopically negative samples and with dead mosquitoes as follows: up to day 5 post-infection (dpi) with abdomens only, after $7 \mathrm{dpi}$ on all negative abdomens, thoraces and heads. Pools were used if there were more than three samples of the same kind on the same day of infection.

\section{Data analysis}

For each trial, feeding rates as well as mortality rates at 1 and 5 dpi and at 14 dpi were calculated. Differences in mortality rates within and between species were calculated in a $2 \times 2$ contingency table using Fisher's exact test with two-tailed $P$-values on GraphPad Software (www.graphpad.com); P-values below 0.05 were considered as statistically significant.

The positivity rate of the mosquitoes was calculated as the percentage of blood-fed mosquitoes that had any developmental stage and/or positive PCR result out of all blood-fed mosquitoes.

The following indices of experimental filarial infections were calculated based on previous publications [31, 42]
The end of the incubation period was set at $10 \mathrm{dpi}$, as this equals the theoretical extrinsic incubation time at $27{ }^{\circ} \mathrm{C}$ according to DDUs [22]. If L3 larvae appeared before $10 \mathrm{dpi}$, calculations were based on the first appearance of L3.

Additionally to the above mentioned calculations, the first appearance of motile L3 in the proboscis was taken into account to assess the vector competence.

\section{Results Infection trial}

Mosquitoes of laboratory strains of Ae. japonicus ( $n=$ 65), Ae. geniculatus $(n=35)$ and Ae. aegypti $(n=105)$ as well as field-collected Ae. japonicus $(n=151)$ were allowed to feed through Parafilm ${ }^{\odot}$ membranes on blood containing microfilariae of $D$. immitis or $D$. repens, or on negative control blood (Ae. japonicus groups only) (Table 1 ). The feeding rates were around $50 \%$ for all Ae. aegypti and Ae. japonicus except in one treatment (24\%, field-collected Ae. japonicus feeding on $D$. repens blood, Table 1), and around $70 \%$ for the Ae. geniculatus groups.

Cumulative mortality rates of naturally dead mosquitoes were calculated from the total number of blood-fed

Infection rate IR $=\frac{\text { number of blood-fed mosquitoes with L3 in body }}{\text { surviving mosquitos at end of incubation period }} \times 100$

Vector efficiency index VEI $=\frac{\text { average number of L3 in mosquitos from end of incubation period to end of study }}{\text { average number of ingested microfilariae }}$ 
Table 2 Specimens of laboratory colony Aedes japonicus PEN $(n=10)$ examined microscopically for larval stages of Dirofilaria immitis

\begin{tabular}{|c|c|c|c|c|c|c|c|}
\hline \multirow[b]{2}{*}{ Dpi } & \multicolumn{2}{|c|}{ Mosquitoes } & \multicolumn{4}{|c|}{ Number of larval stages per individual positive mosquito } & \multirow[b]{2}{*}{ Location (total no. in all dissected mosquitoes) ${ }^{\mathrm{b}}$} \\
\hline & Total no. & No. positive & $\overline{\mathrm{mf}}$ & L1 & $\mathrm{L} 2$ & L3 & \\
\hline 1 & 2 & $1^{\mathrm{a}}$ & 2 & - & - & - & Midgut (2) \\
\hline 3 & 3 & $2^{\mathrm{a}}$ & - & 1,3 & - & - & Malpighian tubules (4) \\
\hline 4 & 1 & $0^{\mathrm{a}}$ & - & - & - & - & \\
\hline 5 & 2 & $1^{\mathrm{a}}$ & 1 & 11 & - & - & Malpighian tubules (11, of which 3 melanized) \\
\hline 6 & 1 & $0^{\mathrm{a}}$ & - & - & - & - & \\
\hline 14 & 1 & 0 & - & - & - & - & \\
\hline
\end{tabular}

mosquitoes in comparison to those that died until 1 and 5 dpi and until 14 dpi. Mosquitoes taken alive for dissection were not considered as dead mosquitoes but were assumed to have lived until $14 \mathrm{dpi}$ (Table 1). At $1 \mathrm{dpi}$, mortality only occurred in the Ae. aegypti IPNC group; this was statistically significantly different to the mortality in field-collected Ae. japonicus infected with $D$. immitis $(P=0.046)$. No other statistically significant differences were observed within the $D$. immitis experiments between the different species at 5 and at $14 \mathrm{dpi}$.

Until $5 \mathrm{dpi}$, there was no mortality in the $D$. repens inoculated groups, except in Ae aegypti IPNC. The mortality on $5 \mathrm{dpi}$ in the $D$. repens inoculated groups was significantly different between Ae. aegypti IPNC and Ae. geniculatus $(P=0.0299)$ and field collected Ae. japonicus $(P=0.0047)$. Mortality rates varied between 0 and $80 \%$, and were usually around $40 \%$ or higher at $14 \mathrm{dpi}$, including the control mosquitoes. The exception was the field-collected Ae. japonicus population inoculated with $D$. repens with an overall mortality rate of $0 \%$. This was significantly different to the mortality in other $D$. repens infected groups $(P=0.0361$ for Ae. japonicus PEN, $P=0.0211$ for Ae. geniculatus and $P=0.01$ for Ae. aegypti) and significantly different in comparison to this field population infected with $D$. immitis at $5 \mathrm{dpi}$ and at $14 \mathrm{dpi}(P=0.0086$ and 0.0035 , respectively). Only in $A e$. aegypti IPNC was the overall mortality rate observed already at $5 \mathrm{dpi}$, whereas in all other mosquito species mortality increased until at $14 \mathrm{dpi}$ (Table 1).

The calculated infectious dose per mosquito in the trials was $12 \mathrm{mf}$, considering the microfilarial density of $3000 / \mathrm{ml}$ blood and an average blood meal volume of $4 \mu \mathrm{l}$. The observed infectious doses as determined by microscopy at 1 dpi differed considerably (Tables 2, 3, 4, $5,6,7,8$ and 9). For D. repens they varied from 0 to 22 and for $D$. immitis from 0 to 7.

\section{Aedes japonicus (PEN) inoculated with D. immitis or $D$. repens}

Altogether 12 blood-fed mosquitoes were in the trial with Ae. japonicus PEN inoculated with microfilariae of $D$. immitis. Out of these, alive mosquitoes $(n=4)$ or freshly dead mosquitoes $(n=6)$ were dissected (Table 2$)$, and a further 2 dead mosquitoes (desiccated) were analysed by PCR only. Four of the 10 dissected mosquitoes were positive for $D$. immitis in microscopy, and another 5 were positive by PCR only. The 2 dead and desiccated mosquitoes were PCR positive [at $2 \mathrm{dpi}$ (abdomen) and 12 dpi (abdomen and head)]. Thus, 11 out of the total 12 mosquitoes (91.7\%) were positive for D. immitis. Mosquitoes were positive in microscopy until at $5 \mathrm{dpi}$, but by PCR the last positive was found at $12 \mathrm{dpi}$. Figure 1

Table 3 Specimens of laboratory colony Aedes japonicus PEN $(n=8)$ examined for larval stages of Dirofilaria repens

\begin{tabular}{|c|c|c|c|c|c|c|c|}
\hline \multirow[b]{2}{*}{ Dpi } & \multicolumn{2}{|c|}{ Mosquitoes } & \multicolumn{4}{|c|}{ Number of larval stages per individual positive mosquito } & \multirow[b]{2}{*}{ Location (total no. in all dissected mosquitoes) ${ }^{\mathrm{a}}$} \\
\hline & Total no. & No. positive & $\mathrm{mf}$ & L1 & $\mathrm{L} 2$ & L3 & \\
\hline 1 & 1 & 1 & 22 & - & - & - & \\
\hline 3 & 1 & 1 & 1 & - & - & - & \\
\hline 5 & 1 & 1 & - & 1 & - & - & \\
\hline 6 & 2 & 1 & - & 1 & - & - & \\
\hline 12 & 1 & 0 & - & - & - & - & \\
\hline 14 & 1 & 1 & - & - & - & 8 & Proboscis (6), head (1), Malpighian tubules (1) \\
\hline 16 & 1 & 1 & - & - & - & 4 & Head (1), abdomen (3) \\
\hline
\end{tabular}

Abbreviations: $d p i$ day post-inoculation, $m f$ microfilariae, $L 1$ first-stage larva, $L 2$ second-stage larva, $L 3$ third-stage larva (infectious stage)

${ }^{a}$ Data given only when localisation was clearly assignable after dissection 
Table 4 Specimens of Aedes japonicus $(\mathrm{CH})(n=24)$ examined microscopically for larval stages of Dirofilaria immitis

\begin{tabular}{|c|c|c|c|c|c|c|c|}
\hline \multirow[b]{2}{*}{ Dpi } & \multicolumn{2}{|c|}{ Mosquitoes } & \multicolumn{4}{|c|}{ Number of larval stages per individual positive mosquito } & \multirow[b]{2}{*}{ Location (total no. in all dissected mosquitoes) ${ }^{c}$} \\
\hline & Total no. & No. positive & $\overline{\mathrm{mf}}$ & L1 & L2 & L3 & \\
\hline 1 & 3 & 2 & 5,4 & - & - & - & \\
\hline 3 & 6 & 6 & $1,1,1,1$ & $5,4,2,1$ & - & - & \\
\hline 4 & 3 & $1^{\mathrm{b}}$ & - & 6 & - & - & \\
\hline 7 & 3 & 2 & - & 7 & 5,3 & 1 & In Malpighian tubules, L1 melanized \\
\hline 10 & 3 & 2 & - & - & - & 1,6 & In Malpighian tubules (7) \\
\hline 14 & $3^{a}$ & 3 & - & - & 1 & 1,3 & Head (1), Malpighian tubules (2) \\
\hline 21 & 3 & 3 & - & - & 1 & $1,4,9$ & Proboscis (7), head (1), thorax (5) \\
\hline
\end{tabular}

Abbreviations: dpi day post-inoculation, $m f$ microfilariae, $L 1$ first-stage larva, $L 2$ second-stage larva, $L 3$ third-stage larva (infectious stage)

aProboscis of one mosquito was lost during the dissection process

${ }^{\mathrm{b}}$ Additional two positive in PCR only

'Data given only when localisation was clearly assignable after dissection

shows L1 larvae (alive and melanized) in the Malpighian tubules and a melanized microfilaria at $5 \mathrm{dpi}$. As no L3 of $D$. immitis were observed, both IR and VEI for $D$. immitis in Ae. japonicus PEN were 0\%. However, the head of a dead mosquito was PCR-positive at 12 dpi. Nonetheless, Ae. japonicus PEN may be refractory to infection with $D$. immitis

Eight blood-fed mosquitoes were in the trial with Ae. japonicus $\mathrm{PEN}$ inoculated with microfilariae of $D$. repens (Table 3), and all mosquitoes were dissected (6 alive and 2 freshly dead). Six of the 8 mosquitoes were positive for D. repens in microscopy (75\%), and no additional one was identified positive by PCR. First L3 larvae were observed at $14 \mathrm{dpi}$ in abdomen and proboscis (Table 3). The IR for D. repens was calculated to be $33.3 \%$ and the VEI $18.2 \%$. Thus, Ae. japonicus PEN is susceptible to infection with $D$. repens.

\section{Aedes japonicus $\mathrm{CH}$ inoculated with $D$. immitis or $D$. repens}

Thirty-one blood-fed mosquitoes were in the trial with Ae. japonicus $\mathrm{CH}$ inoculated with microfilariae of $D$. immitis, and 24 mosquitoes were dissected (18 alive and 6 freshly dead; see Table 4). Nineteen were positive for D. immitis, and an additional 2 were identified positive by PCR. Five of 7 further dead and desiccated mosquitoes were positive for $D$. immitis DNA in their abdomens at 2, 7, 8 and $10 \mathrm{dpi}$. Thus, altogether 26 out of total 31 (83.9\%) mosquitoes were positive for D. immitis. Various developmental stages are shown in Fig. 2.

Seventeen mosquitoes took a blood meal containing microfilariae of $D$. repens (Table 5 ). There were no mortalities, and all were dissected. A total of 16 (94.1\%) were positive for $D$. repens. The only microscopically negative mosquito was also negative by PCR. Different developmental stages are shown in Fig. 3.

First L3 larvae of D. immitis were observed at $14 \mathrm{dpi}$ in the proboscis and of $D$. repens at $10 \mathrm{dpi}$. L3 of both Dirofilaria species were found until the end of the experiments at $21 \mathrm{dpi}$.

The IR and VEI were 27.8 and $66.7 \%$ for $D$. immitis, and 47.1 and $85.9 \%$ for D. repens, respectively, rendering Ae. japonicus $\mathrm{CH}$ a susceptible vector for both filarial species.

\section{Aedes geniculatus IPZ inoculated with $D$. repens or $D$. immitis}

Thirteen blood-fed mosquitoes were in the trial with $A e$. geniculatus IPZ inoculated with microfilariae of $D$. immitis, and 9 alive mosquitoes were dissected (Table 6).

Table 5 Specimens of Aedes japonicus $(\mathrm{CH})(n=17)$ examined for larval stages of Dirofilaria repens

\begin{tabular}{|c|c|c|c|c|c|c|c|}
\hline \multirow[b]{2}{*}{ Dpi } & \multicolumn{2}{|c|}{ Mosquitoes } & \multicolumn{4}{|c|}{ Number of larval stages per individual positive mosquito } & \multirow[b]{2}{*}{ Location (total no. in all dissected mosquitoes) ${ }^{a}$} \\
\hline & Total no. & No. positive & Mf & L1 & $\mathrm{L} 2$ & L3 & \\
\hline 1 & 5 & 4 & $19,20,8,1$ & - & - & - & \\
\hline 4 & 2 & 2 & - & 11,18 & - & - & \\
\hline 7 & 2 & 2 & - & & 23,9 & - & L2 in Malpighian tubules (32) \\
\hline 10 & 3 & 3 & - & - & 4,1 & $6,3,12$ & Proboscis (9), thorax (9), abdomen (4), Malpighian tubules (4) \\
\hline 14 & 3 & 3 & - & 1,5 & 7 & $8,11,11$ & proboscis (26), head (1), abdomen (3) \\
\hline 21 & 2 & 2 & - & - & - & 12,3 & Proboscis (13), abdomen (2) \\
\hline
\end{tabular}

Abbreviations: $d p i$ day post-inoculation, $m f$ microfilariae, $L 1$ first-stage larva, $L 2$ second-stage larva, $L 3$ third-stage larva (infectious stage)

${ }^{a}$ Data given only when localisation was clearly assignable after dissection 
Table 6 Specimens of laboratory colony Aedes geniculatus IPZ $(n=9)$ examined microscopically for larval stages of Dirofilaria immitis

\begin{tabular}{|c|c|c|c|c|c|c|c|}
\hline \multirow[b]{2}{*}{ Dpi } & \multicolumn{2}{|c|}{ Mosquitoes } & \multicolumn{5}{|c|}{ Number of larval stages per individual positive mosquito } \\
\hline & Total no. & No. positive & Mf & L1 & $\mathrm{L} 2$ & L3 & Location (total no. in all dissected mosquitoes) $^{c}$ \\
\hline 1 & 2 & 2 & 5,6 & - & - & - & \\
\hline 4 & 2 & $0^{\mathrm{a}}$ & - & - & - & - & \\
\hline 7 & 2 & $2^{\mathrm{b}}$ & - & 25 & - & - & Malphighian tubules (24), melanized in Malpighian tubules (1) \\
\hline 9 & 0 & $0^{\mathrm{b}}$ & - & - & - & - & \\
\hline 10 & 2 & $1^{\mathrm{b}}$ & - & - & - & 11 & Malpighian tubules (11) \\
\hline 13 & 1 & 1 & - & - & - & 6 & Abdomen (5), Thorax (1) \\
\hline
\end{tabular}

Abbreviations: $d p i$ day post-inoculation, $m f$ microfilariae, $L 1$ first-stage larva, $L 2$ second-stage larva, $L 3$ third-stage larva (infectious stage)

${ }^{\text {a }}$ Two additional positive in PCR only

${ }^{\mathrm{b}}$ One additional positive in PCR only

'Data given only when localisation was clearly assignable after dissection

Six of them were positive in microscopy for D. immitis, and an additional 3 were identified positive by PCR. Furthermore 3 of 4 dead and desiccated mosquitoes was positive for $D$. immitis DNA in their abdomens at 4,7 and 9 dpi. Thus, altogether 12/13 (92.3\%) mosquitoes were positive for $D$. immitis.

Twelve mosquitoes took a blood meal containing microfilariae of $D$. repens. Eight were dissected and were positive in microscopy (Table 7). One out of 4 dead and desiccated mosquitoes was also PCR positive. Thus, 9 out of $12(75 \%)$ mosquitoes were positive for D. repens. Figure 4 shows a massive infection of Malpighian tubules with L2 and L3 larvae at 9 dpi.

First L3 larvae of $D$. immitis were observed at $10 \mathrm{dpi}$ in the abdomen and at 13 dpi also in the thorax. Dirofilaria repens L3 first occurred at 9 dpi in Malpighian tubules and at $14 \mathrm{dpi}$ in the proboscis.

The IR and VEI were 22.2 and $100 \%$ for D. immitis, and 37.5 and $70.8 \%$ for $D$. repens, respectively. Thus, Ae. geniculatus IPZ is susceptible to both filarial species.

Aedes aegypti IPNC inoculated with $D$. repens or $D$. immitis Altogether 24 blood-fed mosquitoes were in the trial with Ae aegypti IPCN inoculated with microfilariae of D. immitis (Table 8 ), and 22 (14 alive and 8 freshly dead) were dissected. Eight were positive for $D$. immitis in microscopy and an additional 9 were positive by PCR on DNA from the abdomens (up to $16 \mathrm{dpi}$ ). Two further dead and desiccated specimens were negative by PCR.
Therefore, a total of $17 / 24(70.8 \%)$ mosquitoes were positive.

Nineteen blood-fed mosquitoes were in the trial with Ae. aegypti IPCN inoculated with microfilariae of $D$. repens (Table 9) and 18 (12 alive and 6 freshly dead) were dissected. Six were positive for $D$. repens in microscopy and an additional 3 by PCR (up to day 9). A total of 9 out of 19 (47.4\%) mosquitoes were positive.

Microfilariae and L1 larvae could only be observed in microscopy until at $5 \mathrm{dpi}$, and no developmental stages were observed after day 5 for both filarial species. As no L3 developed, infection rate and vector efficiency index were $0 \%$ for both $D$. immitis and D. repens. Therefore, according to our study, Ae. aegypti IPNC is refractory to infection with both filarial species.

\section{Development of larval stages}

Infected Malpighian tubules had sac-like appearance and developmental stages were generally found in the distal part of the tubules, as has been described previously [22]. In none of the mosquitoes were all tubules infected. The length and width of the observed larval stages in the mosquitoes are shown in Tables 10 and 11 . Generally, fairly large variations in the sizes of the developmental stages were observed. Additionally, melanized larval stages were observed: for D. immitis in Ae. japonicus PEN at 5 dpi (microfilaria: $305 \times 8 \mu \mathrm{m}$; L1: $164 \times 22 \mu \mathrm{m}$ ) and in Ae. japonicus $\mathrm{CH}$ at $7 \mathrm{dpi}(\mathrm{L} 1: 157 \times 24 \mu \mathrm{m} ; 173 \times 24 \mu \mathrm{m}$;

Table 7 Specimens of laboratory colony Aedes geniculatus IPZ $(n=8)$ examined microscopically for larval stages of Dirofilaria repens

\begin{tabular}{|c|c|c|c|c|c|c|c|}
\hline \multirow[b]{2}{*}{ Dpi } & \multicolumn{2}{|c|}{ Mosquitoes } & \multicolumn{4}{|c|}{ Number of larval stages per individual positive mosquito } & \multirow[b]{2}{*}{ Location (total no. in all dissected mosquitoes) ${ }^{a}$} \\
\hline & Total no. & No. positive & $\overline{\mathrm{Mf}}$ & L1 & L2 & L3 & \\
\hline 1 & 3 & 3 & $6,8,20$ & - & - & - & \\
\hline 5 & 2 & 2 & - & 3,7 & - & - & Malpighian tubules (3) \\
\hline 9 & 1 & 1 & - & 3 & 19 & 14 & Malphighian tubules (36) \\
\hline 14 & 2 & 2 & - & 1 & - & 1,25 & L3: Proboscis (20), thorax (2), Malpighian tubules (4) \\
\hline
\end{tabular}

Abbreviations: $d p i$ day post-inoculation, $m f$ microfilariae, $L 1$ first-stage larva, $L 2$ second-stage larva, $L 3$ third-stage larva (infectious stage)

${ }^{a}$ Data given only when localisation was clearly assignable after dissection 
Table 8 Specimens of laboratory colony Aedes aegypti IPNC $(n=22)$ examined microscopically for larval stages of Dirofilaria immitis

\begin{tabular}{|c|c|c|c|c|c|c|}
\hline \multirow[b]{2}{*}{ Dpi } & \multirow[b]{2}{*}{ Total no. of individuals } & \multirow[b]{2}{*}{ No. of positive individuals } & \multicolumn{4}{|c|}{ Number of larval stages per individual positive mosquito ${ }^{c}$} \\
\hline & & & $\mathrm{mf}$ & L1 & L2 & L3 \\
\hline 1 & 9 & $5^{a}$ & $1,1,5,7,10$ & - & - & - \\
\hline 2 & 1 & 1 & - & 1 & - & - \\
\hline 3 & 4 & $1^{\mathrm{b}}$ & 1 & - & - & - \\
\hline 5 & 2 & 1 & - & 3 & - & - \\
\hline 7 & 2 & $0^{\mathrm{a}}$ & - & - & - & - \\
\hline 9 & 1 & $0^{b}$ & - & - & - & - \\
\hline 12 & 1 & $0^{b}$ & - & - & - & - \\
\hline 14 & 1 & $0^{b}$ & - & - & - & - \\
\hline 16 & 1 & $0^{b}$ & - & - & - & - \\
\hline
\end{tabular}

Abbreviations: $d p i$ day post-inoculation, $m f$ microfilariae, $L 1$ first-stage larva, $L 2$ second-stage larva, $L 3$ third-stage larva (infectious stage)

${ }^{\text {a }}$ Two additional positive in PCR

${ }^{\mathrm{b}}$ One additional positive in PCR only

${ }^{\mathrm{C}}$ Localisation of developmental stages was not clearly assignable after dissection

$187 \times 25 \mu \mathrm{m})$, and for $D$. repens in Ae. japonicus $\mathrm{CH}$ at 14 dpi (L1: $177 \times 26 \mu \mathrm{m})$.

\section{Discussion}

More than 60 mosquito species are incriminated vectors of Dirofilaria spp., and several species have been examined under laboratory conditions for their potential vector competence by observing the development to the infective L3 stage. For D. immitis these include for example Ae. aegypti [40, 43-45], Ae. albopictus [46-48], Ae. koreicus [31], Aedes vexans (Meigen, 1830) [22] and Aedes triseriatus Say, 1823 [49], and for D. repens e.g. Ae. aegypti [45, 50-52], Ae. albopictus [46], Ae. vexans, Aedes mariae (Sergent \& Sergent, 1903) [52], Anopheles stephensi Liston, 1901 [45], Anopheles. maculipennis atroparvus Wellcome, 1901 [45] and Culex pipiens molestus Forskål, 1775 [51].

To our knowledge, here we could demonstrate for the first time that field-collected Ae. japonicus are susceptible vectors for both Dirofilaria species. For example, the VEI for $D$. immitis was $66.7 \%$ which is distinctly higher than the corresponding values estimated for two other invasive Aedes species, Ae albopictus and Ae. koreicus [31]. In addition, high abundances of Ae. japonicus have been reported from several introduction sites both in Europe and Northern America [32, 53-56]. Further, Ae. japonicus was shown to readily feed on mammals including humans and dogs [56]. Taken together, our findings suggest that there is an increased risk of Dirofilaria transmission in areas populated by this species. This is somewhat reminiscent to the situation in Italy where the establishment of $\mathrm{Ae}$. albopictus, a suitable vector of $D$. immitis, changed the epidemiology of canine dirofilarioses (transmission in new areas, higher prevalences) [26, 27].

Interestingly, the laboratory colony of Ae. japonicus PEN was susceptible to $D$. repens but seemed refractory to $D$. immitis, i.e. no L3 larvae developed and reached the proboscis. Though we only had few blood-engorged

Table 9 Specimens of laboratory colony Aedes aegypti IPNC $(n=18)$ examined microscopically for larval stages of Dirofilaria repens

\begin{tabular}{|c|c|c|c|c|c|c|}
\hline \multirow[b]{2}{*}{ Dpi } & \multirow[b]{2}{*}{ Total no. of individuals } & \multirow[b]{2}{*}{ No. of positive individuals } & \multicolumn{4}{|c|}{ Number of larval stages per individual positive mosquito ${ }^{c}$} \\
\hline & & & Mf & L1 & L2 & L3 \\
\hline 1 & 6 & $3^{\mathrm{a}}$ & $1,3,6$ & - & - & - \\
\hline 3 & 4 & 2 & 16,2 & - & - & - \\
\hline 5 & 2 & 1 & - & 1 & - & - \\
\hline 7 & 2 & $0^{a}$ & - & - & - & - \\
\hline $9^{b}$ & 1 & $0^{a}$ & - & - & - & - \\
\hline 12 & 1 & 0 & - & - & - & - \\
\hline 14 & 1 & 0 & - & - & - & - \\
\hline 16 & 1 & 0 & - & - & - & - \\
\hline
\end{tabular}

Abbreviations: $d p i$ day post-inoculation, $m f$ microfilariae, $L 1$ first-stage larva, $L 2$ second-stage larva, $L 3$ third-stage larva (infectious stage)

${ }^{\mathrm{a}}$ One additional positive in PCR only

${ }^{\mathrm{b}} \mathrm{Head}$ was lost during the dissection process

'Localisation of developmental stages was not clearly assignable after dissection 
Table 10 Sizes of developmental stages of Dirofilaria repens reared under laboratory conditions at $27^{\circ} \mathrm{C}$

\begin{tabular}{llllll}
\hline Days post-infection & Measurement $(\mu \mathrm{m})$ & Ae. japonicus PEN & Ae. japonicus CH & Ae. geniculatus IPZ & Ae. aegypti IPNC \\
\hline $1-2$ & Mean length (range) & $331(309-350)$ & - & $237(221-254)$ & - \\
& Mean width (range) & $8(5-10)$ & - & $6(5-6)$ & - \\
$3-6$ & Mean length (range) & $208(144-272)$ & - & - & - \\
& Mean width (range) & $25(21-30)$ & - & $735(313-361)$ \\
$7-9$ & Mean length (range) & $263(\mathrm{na})$ & $602(535-678)$ & - \\
& Mean width (range) & $283(\mathrm{na})$ & $30(23-34)$ & $35(10-48)$ & - \\
$10-14$ & Mean length (range) & $954(822-1030)$ & $822(685-954)$ & $911(657-1120)$ & - \\
& Mean width (range) & $26(23-28)$ & $24(22-27)$ & $29(26-36)$ & - \\
More than 14 & Mean length (range) & $976(\mathrm{na})$ & - & - & - \\
& Mean width (range) & 27 (na) & - & - \\
\hline
\end{tabular}

Abbreviations: na not applicable (only single specimens available); -, no specimens available or no photographs taken during dissection process

females of this mosquito strain in this trial, this finding emphasizes again the need to carry out vector competence experiments with local and wild specimens to obtain relevant results. Unfortunately, the experiment cannot be repeated due to loss of the colony.

Additionally we could show that a laboratory colony of Ae. geniculatus derived from field-collected individuals [37] is an equally good vector for both Dirofilaria species. The univoltine Ae. geniculatus shares larval breeding sites such as tree holes with the invasive Ae. japonicus [37]. It generally occurs in low abundances but large numbers may be present in focal areas. Taking into account its aggressive mammophilic biting behaviour, Ae. geniculatus may contribute to local transmission cycles. Further, our established colony might be of value for further studies on host-pathogen interactions.

Constant temperatures of $27{ }^{\circ} \mathrm{C}$ are not realistic for central Europe, though the average temperature might reach this level during hot summer spells. Temperatures fluctuating over the day and between days are reality for the climate in central Europe, and further investigations will be done at more realistic fluctuating temperature regimes. Interestingly, daily temperature fluctuations accelerated pathogen development in the mosquito as compared to constant conditions with the same average temperature, as was shown with Plasmodium parasites, D. immitis and dengue viruses [57-59], and this was particularly observed under cool conditions which is of significance at the cooler margin of a suitable climate.

The developmental time from microfilariae to infectious L3 stage was in accordance with the predictions from the DDU formula. The experiments in this study were done at constant $27^{\circ} \mathrm{C}$; at this temperature, the development time is expected to be 10 days until the first observation of L3. However, even though first L3 larvae were observed within 10 days in Ae. japonicus and Ae. geniculatus, they tended to reach the proboscis only a few days later, and this has to be taken into account additionally when making a risk assessment.

In the first $2 \mathrm{dpi}, D$. repens $\mathrm{L} 1$ stages started to shorten in length, but remained at the same approximate width as microfilariae as compared to measurements described in literature [60]. After 3-6 days both D. repens and $D$. immitis had reached the typical sausage stage $[39,40]$ in

Table 11 Sizes of developmental stages of Dirofilaria immitis reared under laboratory conditions at $27^{\circ} \mathrm{C}$

\begin{tabular}{llllll}
\hline Day post-infection & Measurement $(\mu \mathrm{m})$ & Ae. japonicus PEN & Ae. japonicus CH & Ae. geniculatus IPZ & Ae. aegypti IPNC \\
\hline $1-2$ & Mean length (range) & - & - & - & $-283(256-290)$ \\
& Mean width (range) & - & - & - & $8(6-10)$ \\
$4-6$ & Mean length (range) & $166(122-215)$ & $218(171-250)$ & - & $260(244-270)$ \\
& Mean width (range) & $22(14-35)$ & $25(23-28)$ & - & $3.9(5.5-6.2)$ \\
$7-9$ & Mean length (range) & - & $284(170-353)$ & $359(283-418)$ & - \\
& Mean width (range) & - & $28(25-33)$ & $32(28-35)$ & - \\
$10-14$ & Mean length (range) & - & $859(477-922)$ & $811(552-1050)$ & - \\
& Mean width (range) & - & $33(27-36)$ & $29(23-37)$ & - \\
More than 14 & Mean length (range) & - & $1022(906-1130)$ & - & - \\
& Mean width (range) & - & $29(25-32)$ & - & - \\
\hline
\end{tabular}




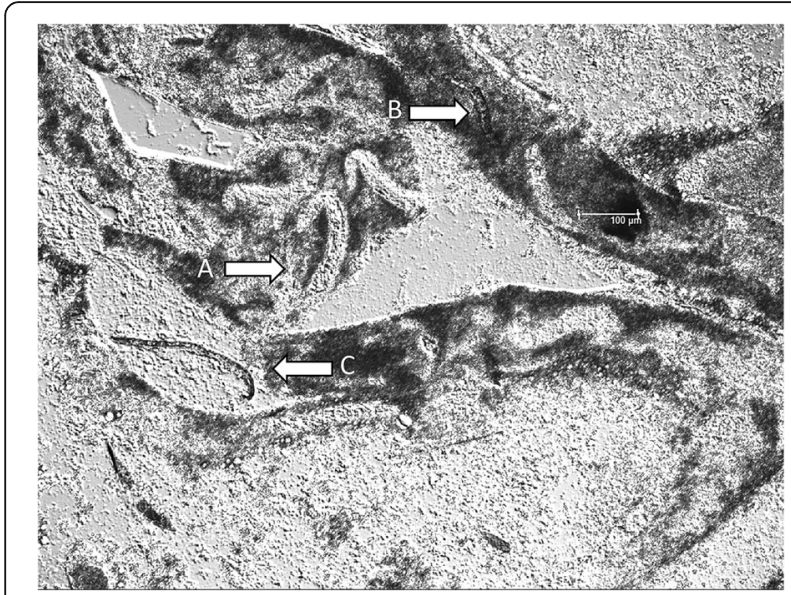

Fig. 1 Alive (a) and melanised (b) first-stage larvae (L1) and melanised microfilaria (c) of Dirofilaria immitis in Malpighian tubules of Aedes japonicus PEN at 5 dpi
Ae. japonicus, whereas they had only marginally shortened in the refractory Ae. aegypti IPNC, indicating that development did hardly take place in this mosquito species, and microfilariae most probably died in these first days post-inoculation. Aedes aegypti has been considered a rather unsuitable natural host for $D$. immitis, but a large variability of its susceptibility has been reported on many occasions $[40,43,44,61]$. Both $D$. immitis and $D$. repens larvae started to elongate to L2 stage after 6 days at sizes comparable to what has been described earlier [40]. After 14 days and later, the infectious $D$. immitis L3 larvae reached their full length [40], with a lower size variation than described previously [22]. Interestingly, several larval stages of $D$. repens observed also in thorax and proboscis were shorter than previously described for L3 stages, and many of them were under $1000 \mu \mathrm{m}$. Previously, it was reported that the infectious stages reached lengths above $1000 \mu \mathrm{m}$ [39].
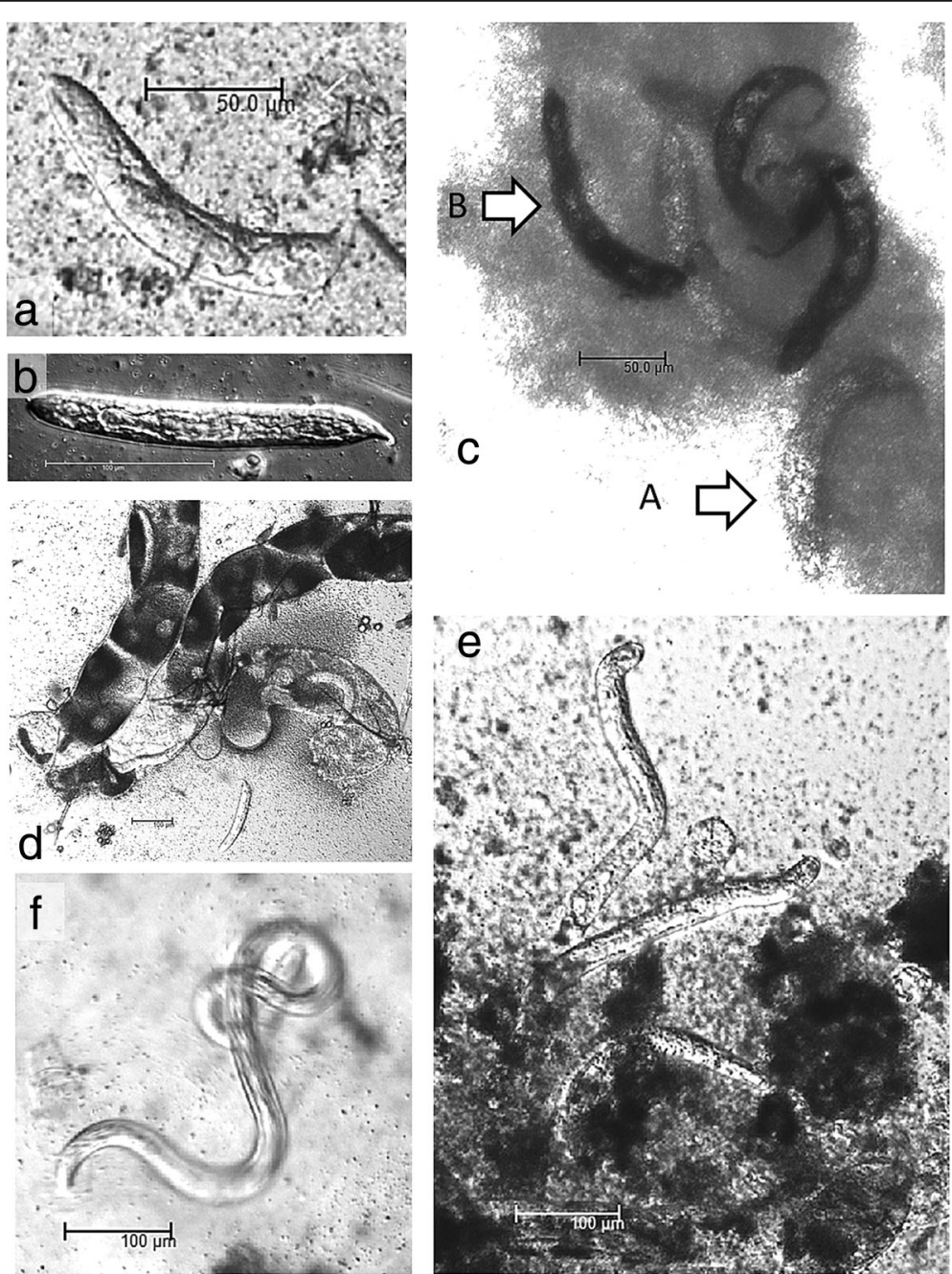

Fig. 2 Developmental stages of Dirofilaria immitis in Aedes japonicus collected from the field in Switzerland: a, b L1 larvae at 4 dpi. c L1 alive (A) and melanised (B) larvae at 7 dpi. $\mathbf{d} L 1$ larvae in Malpighian tubules at 4 dpi. e L2 larvae at 7 dpi. $\mathbf{f} L 3$ larvae at 14 dpi 

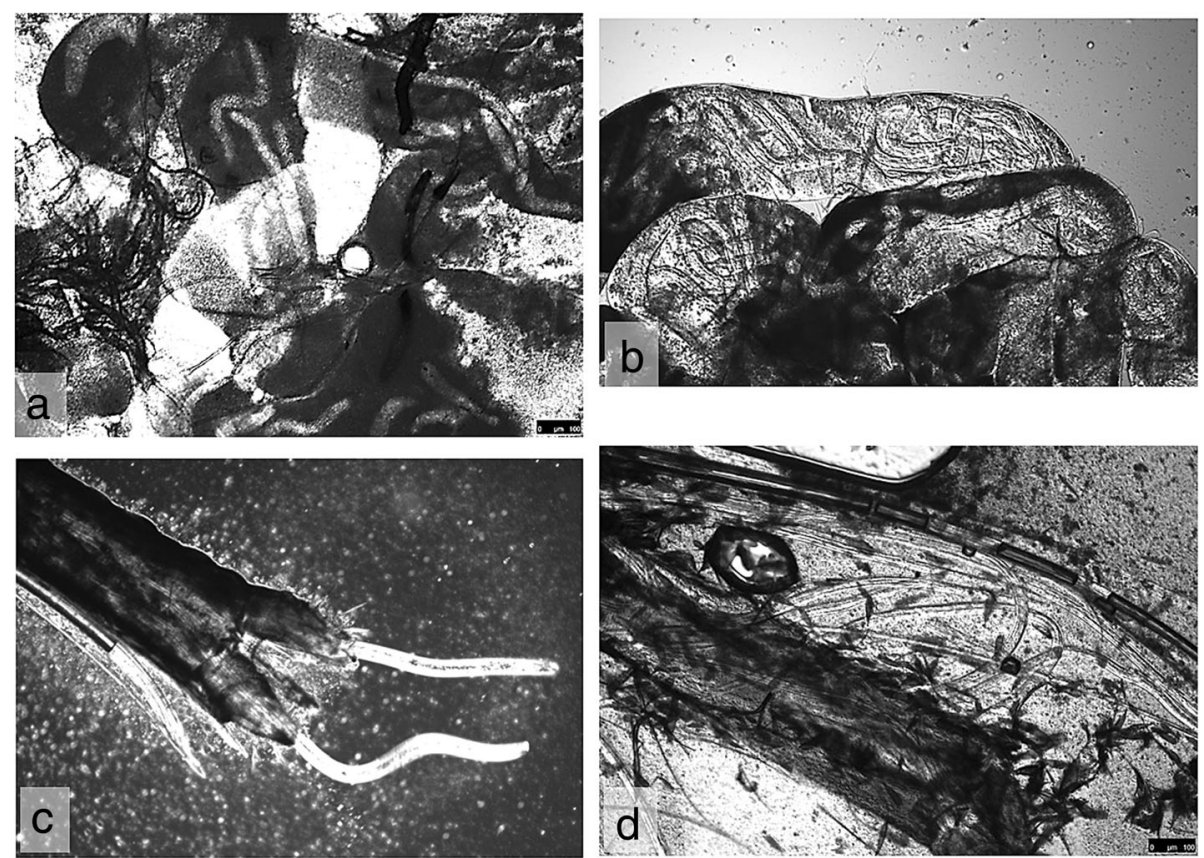

Fig. 3 Developmental stages of Dirofilaria repens in Aedes japonicus collected from the field in Switzerland: a Different developmental stages, alive and melanized, in Malpighian tubules at 14 dpi. b L2, and possibly L3 larvae, in Malpighian tubules at 7 dpi. c, d L3 larvae at 14 and 21 dpi, respectively

Microscopy is a good and sensitive tool to observe the developmental stages of Dirofilaria spp. in mosquito samples. However, especially in the early stage of infection, additional positive specimens can be detected by PCR when low numbers of microfilariae originating from the infectious blood meals may be overlooked. In addition, PCRs remained positive until the end of the trials although development ceased at $5 \mathrm{dpi}$ for D. immitis in Ae. japonicus PEN, and in Ae. aegypti IPNC for both filarial species. Thus, PCR positivity gives no clue whatsoever

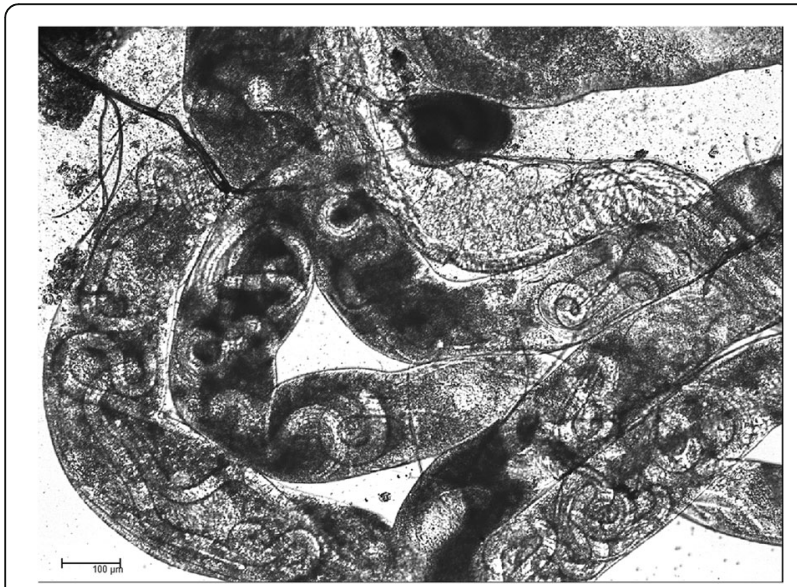

Fig. $4 \mathrm{~L} 2$ and $\mathrm{L} 3$ larvae of Dirofilaria repens in Malpighian tubules of Aedes geniculatus (laboratory strain IPZ) at 9 dpi about infection rates and vector efficiency of any mosquito species, but can merely be of use in epidemiological studies to make a general assessment of occurrence of a filarial species in a certain area. This means that DNA reports from field-collected mosquitoes need to be very critically assessed.

We observed a large variation of microfilaria in the blood meals at 1 dpi (Tables 2, 3, 4, 5, 6, 7, 8 and 9). Furthermore, altogether only $75-94 \%$ of the engorged mosquitoes harboured microfilariae at all. This has to be taken into account additionally when making risk assessments. The calculations of VEI are based on the observed number of microfilariae at the beginning of the trial. Due to the low numbers of mosquitoes involved in some experiments, only between 1 and 3 mosquitoes could be used to determine the number of ingested microfilariae. Thus, the obtained VEI values have therefore to be treated with some caution. However, several mosquito species had L3 larvae in the proboscis and those can as such be seen as suitable vectors for the respective filarial species.

A crucial factor for vector competence and vector capacity is that mosquitoes need to survive the extrinsic incubation period in order to be able to transmit the pathogen to the next host. The results indicate that the parasite does not cause an overall higher mortality in the mosquitoes at the infection dose of $3000 \mathrm{mf} / \mathrm{ml}$, which was chosen because it was shown to be suitable for such investigations in previous studies [31]. Higher mortalities 
with inocula containing higher microfilaraemiae were observed in various studies [31, 43, 48]. In endemic foci, very high microfilaraemiae can be observed (up to $70,000 / \mathrm{ml}$ ) [31], and it was speculated that dogs with low microfilaraemiae might be the relevant reservoirs for Dirofilaria transmission [48].

\section{Conclusions}

To our knowledge, field-collected Ae. japonicus were for the first time shown to be an efficient vector for both $D$. immitis and $D$. repens, indicating that this invasive and locally highly abundant species may contribute to a local transmission of filarial worms, as has been described for Ae. albopictus and D. immitis in Italy. Additionally, also the indigenous Ae. geniculatus, sharing the same larval breeding sites with Ae. japonicus, is a suitable vector for Dirofilaria spp. Aedes japonicus from a laboratory colony were refractory to $D$. immits, confirming the necessity to perform vector competence studies and risk assessments based on such studies with local mosquito populations. Our results further demonstrate that by DNA detection alone no reliable conclusions can be drawn with regard to the vector competence of a mosquito species.

\section{Abbreviations}

DDU: Dirofilaria development unit; DIC: differential interference contrast; dpi: day post-infectionem; HDU: heartworm development unit; IR: infection rate; L1: first-stage larva; L2: second-stage larva; L3: third-stage larva; mf: microfilaria; PBS: phosphate buffered saline; VEl: vector efficiency index

\section{Acknowledgments}

The authors would like to thank Jeannine Hauri, Chiara Tochtermann and Christian Bünzli for excellent technical assistance. Eva Veronesi is acknowledged for her advice on experimental mosquito infection and dissection. The work of AM, GC, RB, and CS was done under the frame of COST action TD1303.

\section{Funding}

Not applicable.

\section{Availability of data and materials}

The data supporting the conclusions of this article are included within the article. The datasets used and/or analysed during the current study are available from the corresponding author on reasonable request.

\section{Authors' contributions}

CS and AM had the idea for the study. CS set up the experimental design, performed the experimental infection, laboratory analysis and analysed the data. RB provided study material. GC and FM helped with the experimental design and gave valuable scientific input. CS wrote the manuscript. All authors critically revised the manuscript, read and approved the final manuscript.

\section{Competing interests}

The authors declare that they have no competing interests.

Consent for publication

Not applicable.

Ethics approval

Not applicable.

\section{Author details}

${ }^{1}$ National Centre for Vector Entomology, Institute of Parasitology, Vetsuisse Faculty, University of Zurich, Zurich, Switzerland. ${ }^{2}$ Department for Bacteriology and Parasitology, Croatian Veterinary Institute, Zagreb, Croatia. ${ }^{3}$ Laboratory of Parasitology, Istituto Zooprofilattico Sperimentale delle Venezie, Legnaro, Italy.

Received: 15 December 2016 Accepted: 7 February 2017

Published online: 20 February 2017

\section{References}

1. Simon F, Siles-Lucas M, Morchon R, Gonzalez-Miguel J, Mellado I, Carreton E, et al. Human and animal dirofilariasis: the emergence of a zoonotic mosaic. Clin Microbiol Rev. 2012;25:507-44.

2. Genchi C, Kramer LH, Rivasi F. Dirofilarial infections in Europe. Vector Borne Zoonotic Dis. 2011;11:1307-17.

3. Otranto D, Dantas-Torres F, Brianti E, Traversa D, Petric D, Genchi C, et al. Vector-borne helminths of dogs and humans in Europe. Parasit Vectors. 2013;6:16.

4. Sassnau R, Czajka C, Kronefeld M, Werner D, Genchi C, Tannich E, et al. Dirofilaria repens and Dirofilaria immitis DNA findings in mosquitoes in Germany: temperature data allow autochthonous extrinsic development. Parasitol Res. 2014;113:3057-61.

5. Kronefeld M, Kampen H, Sassnau R, Werner D. Molecular detection of Dirofilaria immitis, Dirofilaria repens and Setaria tundra in mosquitoes from Germany. Parasit Vectors. 2014;7:30.

6. Zielke DE, Schäfer M, Werner D, Kampen H. Mosquito monitoring and surveillance of mosquito-borne pathogens in Germany. In: Tagung der DVGFachgruppe "Parasitologie und parasitäre Krankheiten": 2015. Stralsund: Verlag der DVG Service GmbH; 2015. ISBN: 978-3-86345-261-2. www.dvg.de.

7. Genchi C, Rinaldi L, Mortarino M, Genchi M, Cringoli G. Climate and Dirofilaria infection in Europe. Vet Parasitol. 2009;163:286-92.

8. Sassnau R, Daugschies A, Lendner M, Genchi C. Climate suitability for the transmission of Dirofilaria immitis and D. repens in Germany. Vet Parasitol. 2014;205:239-45.

9. Sassnau R, Genchi C. Qualitative risk assessment for the endemisation of Dirofilaria repens in the state of Brandenburg (Germany) based on temperature-dependent vector competence. Parasitol Res. 2013;112:2647-52.

10. Silbermayr K, Eigner B, Joachim A, Duscher GG, Seidel B, Allerberger F, et al. Autochthonous Dirofilaria repens in Austria. Parasit Vectors. 2014;7:226.

11. Bockova E, Rudolf I, Kocisova A, Betasova L, Venclikova K, Mendel J, et al. Dirofilaria repens microfilariae in Aedes vexans mosquitoes in Slovakia. Parasitol Res. 2013;112:3465-70.

12. Czajka C, Becker N, Jost H, Poppert S, Schmidt-Chanasit J, Kruger A, et al. Stable transmission of Dirofilaria repens nematodes, northern Germany. Emerg Infect Dis. 2014;20:328-31.

13. Löwenstein M, Spallinger E. Erster autochtoner Fall einer Dirofilaria (Nochtiella) repens Infektion bei einem Hund in Österreicht - ein Fallbericht. Wien Tierärztl Mschr - Vet Med Austria. 2009;96:184-7.

14. Overgaauw P, van Dijk E. Autochthonous case of Dirofilaria repens in a dog in the Netherlands. Vet Rec. 2009;164:158.

15. Hermosilla C, Pantchev N, Dyachenko V, Gutmann M, Bauer C. First autochthonous case of canine ocular Dirofilaria repens infection in Germany. Vet Rec. 2006;158:134-5.

16. Pantchev N, Norden N, Lorentzen L, Rossi M, Rossi U, Brand B, et al. Current surveys on the prevalence and distribution of Dirofilaria spp. in dogs in Germany. Parasitol Res. 2009;105S1:S63-74.

17. Sassnau R, Dyachenko V, Pantchev N, Stöckel F, Dittmar K, Daugschies A Dirofilaria repens - Befall in einem Schlittenhunde-Rudel im Land Brandenburg. Diagnose und Therapie der kaninen kutanen Dirofilariose. Tierarztl Prax. 2009;37(K):95-101.

18. Sassnau R, Kohn M, Demeler J, Kohn B, Muller E, Krucken J, et al. Is Dirofilaria repens endemic in the Havelland district in Brandenburg, Germany? Vector Borne Zoonotic Dis. 2013;13:888-91.

19. Tappe D, Plauth M, Bauer T, Muntau B, Diessel L, Tannich E, et al. A case of autochthonous human Dirofilaria infection, Germany, March 2014. Euro Surveill. 2014;19:2-4.

20. Hasler S, Grimm F, Thiel MA, Muller NJ, Eberhard R, Bosch MM. Swiss patient with a subconjunctival Dirofilaria repens. Klin Monbl Augenheilkd. 2010;227:332-3. 
21. Morchon R, Carreton E, Gonzalez-Miguel J, Mellado-Hernandez I. Heartworm disease (Dirofilaria immitis) and their vectors in Europe - New distribution trends. Front Physiol. 2012;3:196.

22. Fortin JF, Slocombe JOD. Temperature requirements for the development of Dirofilaria immitis and Aedes triseriatus and Ae. vexans. Mosquito News. 1981:41:625-33.

23. Genchi C, Mortarino M, Rinaldi L, Cringoli G, Traldi G, Genchi M. Changing climate and changing vector-borne disease distribution: the example of Dirofilaria in Europe. Vet Parasitol. 2011;176:295-9.

24. Masny A, Golab E, Cielecka D, Salamatin R. Vector-borne helminths of dogs and humans - focus on central and eastern parts of Europe. Parasit Vectors. 2013;6:38.

25. Medlock JM, Hansford KM, Schaffner F, Versteirt V, Hendrickx G, Zeller H, et al. A review of the invasive mosquitoes in Europe: ecology, public health risks, and control options. Vector Borne Zoonotic Dis. 2012;12:435-47.

26. Pietrobelli M. Importance of Aedes albopictus in veterinary medicine. Parassitologia. 2008:50:113-5.

27. Giangaspero A, Marangi M, Latrofa MS, Martinelli D, Traversa D, Otranto D, et al. Evidences of increasing risk of dirofilarioses in southern Italy. Parasito Res. 2013;112:1357-61.

28. Bezzhonova OV, Patraman IV, Ganushkina LA, Vyshemirskii Ol, Sergiev VP. The first finding of invasive species Aedes (Finlaya) koreicus (Edwards, 1917) in European Russia. Med Parazitol (Mosk). 2014;1:16-9. In Russian.

29. Montarsi F, Martini S, Dal Pont M, Delai N, Ferro Milone N, Mazzucato M, et al. Distribution and habitat characterization of the recently introduced invasive mosquito Aedes koreicus (Hulecoeteomyia koreica), a new potential vector and pest in north-eastern Italy. Parasit Vectors. 2013;6:292.

30. Versteirt V, De Clercq EM, Fonseca DM, Pecor J, Schaffner F, Coosemans M, et al. Bionomics of the established exotic mosquito species Aedes koreicus in Belgium. Europe J Med Entomol. 2012;49:1226-32.

31. Montarsi F, Ciocchetta S, Devine G, Ravagnan S, Mutinelli F, Frangipane di Regalbono A, et al. Development of Dirofilaria immitis within the mosquito Aedes (Finlaya) koreicus, a new invasive species for Europe. Parasit Vectors. 2015:8:177.

32. Kampen $\mathrm{H}$, Werner D. Out of the bush: the Asian bush mosquito Aedes japonicus japonicus (Theobald, 1901) (Diptera, Culicidae) becomes invasive. Parasit Vectors. 2014;7:59

33. Kaufman MG, Fonseca DM. Invasion biology of Aedes japonicus japonicus (Diptera: Culicidae). Ann Rev Entomol. 2014;59:31-49.

34. Krebs T, Bindler P, L'Ambert G, Toty C, Perrin Y, Jourdain F. First establishment of Aedes japonicus japonicus (Theobald, 1901) (Diptera: Culicidae) in France in 2013 and its impact on public health. J Vector Ecol. 2014;39:437-40

35. Zielke DE, Ibáñez-Justicia A, Kalan K, Merdić E, Kampen H, Werner D. Recently discovered Aedes japonicus japonicus (Diptera: Culicidae) populations in the Netherlands and northern Germany resulted from a new introduction event and from a split from an existing population. Parasit Vectors. 2015;8:40

36. Rishniw M, Barr SC, Simpson KW, Frongillo MF, Franz M, Dominguez Alpizar JL. Discrimination between six species of canine microfilariae by a single polymerase chain reaction. Vet Parasitol. 2006 135:303-14

37. Wagner S, Mathis A. Laboratory colonisation of Aedes geniculatus. J Europ Mos Control Ass. 2016;34:1-4.

38. Friend WG, Smith JJ. Factors affecting feeding by bloodsucking insects. Annu Rev Entomol. 1977;22:309-31.

39. Bain O. Développment en Camargue de la Filaire du Chien, Dirofilaria repens Railliet et Henry, 1911, chez les Aedes halophiles. Bull Mus Natn Hist Nat. 1978;351:19-27.

40. Taylor AE. The development of Dirofilaria immitis in the mosquito Aedes aegypti. J Helminthol. 1960;34:27-38.

41. Albonico F, Loiacono M, Gioia G, Genchi C, Genchi M, Mortarino M. Rapid differentiation of Dirofilaria immitis and Dirofilaria repens in canine peripheral blood by real-time PCR coupled to high resolution melting analysis. Vet Parasitol. 2014;200:128-32.

42. Kartman L. Suggestions concerning an index of experimental filaria infection in mosquitoes. Am J Trop Med Hyg. 1954;3:329-37.

43. Serrao ML, Labarthe N, Lourenco-de-Oliveira R. Vectorial competence of Aedes aegypti (Linnaeus, 1762) Rio de Janeiro strain, to Dirofilaria immitis (Leidy, 1856). Mem Inst Oswaldo Cruz. 2001:96:593-8.
44. Tiawsirisup S, Nithiuthai S. Vector competence of Aedes aegypti (L.) and Culex quinquefasciatus (Say) for Dirofilaria immitis (Leidy). Southeast Asian J Trop Med Public Health. 2006;37S3:110-4.

45. Webber WAF, Hawking F. Experimental maintenance of Dirofilaria repens and D. immitis in dogs. Exp Parasitol. 1955;4:143-64.

46. Cancrini G, Pietrobelli M, Frangipane di Regalbono A, Tampieri MP, Della Torre A. Development of Dirofilaria and Setaria nematodes in Aedes albopictus. Parassitologia. 1995;37:141-5.

47. Nayar JK, Knight JW. Aedes albopictus (Diptera: Culicidae): an experimental and natural host of Dirofilaria immitis (Filarioidea: Onchocercidae) in Florida. USA J Med Entomol. 1999;36:441-8.

48. Lai CH, Tung KC, Ooi HK, Wang JS. Competence of Aedes albopictus and Culex quinquefasciatus as vector of Dirofilaria immitis after blood meal with different microfilarial density. Vet Parasitol. 2000;90:231-7.

49. Intermill RW. Development of Dirofilaria immitis in Aedes triseriatus Say. Mosquito News. 1973;33:176-81.

50. Anyanwu IN, Agbede Rl, Ajanusi OJ, Umoh JU, Ibrahim ND. The incrimination of Aedes (Stegomyia) aegypti as the vector of Dirofilaria repens in Nigeria. Vet Parasitol. 2000;92:319-27.

51. Kuzmin Y, Varodi E, Vasylyk N, Kononko G. Experimental infection of mosquitoes with Dirofilaria repens (Nematoda, Filarioidea) larvae. Vestn Zool. 2005:39:19-24.

52. Cancrini G, Yanchang S, Della Torre A, Coluzzi M. Influenza della Temperatura sullo sviluppo larvale di Dirofilaria repens in diverse specie di zanzare. Parassitologia. 1988;30S1:38.

53. Anderson JF, McKnight S, Ferrandino FJ. Aedes japonicus japonicus and associated woodland species attracted to Centers for Disease Control and Prevention miniature light traps baited with carbon dioxide and the Traptech mosquito lure. J Am MosCon Ass. 2012;28:184-91.

54. Bevins SN. Establishment and abundance of a recently introduced mosquito species Ochlerotatus japonicus (Diptera: Culicidae) in the Southern Appalachians, USA. J Med Entomol. 2007:44:945-52.

55. Schaffner F, Kaufmann C, Hegglin D, Mathis A. The invasive mosquito Aedes japonicus in Central Europe. Med Vet Entomol. 2009;23:448-51.

56. Schönenberger AC, Wagner S, Tuten HC, Schaffner F, Torgerson P, Furrer S, et al. Host preferences of host-seeking and blood-fed mosquitoes in Switzerland. Med Vet Entomol. 2016:30:39-52.

57. Carrington LB, Armijos MV, Lambrechts L. Scott TW. Fluctuations at a low mean temperature accelerate dengue virus transmission by Aedes aegypti. PLoS Negl Trop Dis. 2013;7:e2190.

58. Paaijmans KP, Blanford S, Bell AS, Blanford Jl, Read AF, Thomas MB. Influence of climate on malaria transmission depends on daily temperature variation. Proc Natl Acad Sci USA. 2010;107:15135-9.

59. Ledesma N, Harrington L. Fine-scale temperature fluctuation and modulation of Dirofilaria immitis larval development in Aedes aegypti. Vet Parasitol. 2015;209:93-100

60. Magnis J, Lorentz S, Guardone L, Grimm F, Magi M, Naucke TJ, et al. Morphometric analyses of canine blood microfilariae isolated by the Knott's test enables Dirofilaria immitis and D. repens species-specific and Acanthocheilonema (syn. Dipetalonema) genus-specific diagnosis. Parasit Vectors. 2013;6:48.

61. Sulaiman I. Susceptibility of Aedes aegypti to infections with Dirofilaria immitis and Dirofilaria repens. Southeast Asian J Trop Med Public Health. 1983;14:543-7.

\section{Submit your next manuscript to BioMed Central and we will help you at every step:}

- We accept pre-submission inquiries

- Our selector tool helps you to find the most relevant journal

- We provide round the clock customer support

- Convenient online submission

- Thorough peer review

- Inclusion in PubMed and all major indexing services

- Maximum visibility for your research

Submit your manuscript at www.biomedcentral.com/submit 